\title{
Real-time volumetric MRI thermometry of focused ultrasound ablation in vivo: a feasibility study in pig liver and kidney
}

\author{
Bruno Quesson $^{a *}$, Christophe Laurent ${ }^{a, b}$, Gregory Maclair ${ }^{a}$, Baudouin \\ Denis de Senneville ${ }^{a}$, Charles Mougenot ${ }^{c}$, Mario Ries ${ }^{a}$, Thibault Carteret ${ }^{a}$, \\ Anne Rullier ${ }^{d}$ and Chrit T. W. Moonen ${ }^{\mathrm{a}}$
}

\begin{abstract}
MR thermometry offers the possibility to precisely guide high-intensity focused ultrasound (HIFU) for the noninvasive treatment of kidney and liver tumours. The objectives of this study were to demonstrate therapy guidance by motion-compensated, rapid and volumetric MR temperature monitoring and to evaluate the feasibility of MR-guided HIFU ablation in these organs. Fourteen HIFU sonications were performed in the kidney and liver of five pigs under general anaesthesia using an MR-compatible Philips HIFU platform prototype. HIFU sonication power and duration were varied. Volumetric MR thermometry was performed continuously at $1.5 \mathrm{~T}$ using the proton resonance frequency shift method employing a multi-slice, single-shot, echo-planar imaging sequence with an update frequency of $2.5 \mathrm{~Hz}$. Motion-related suceptibility artefacts were compensated for using multi-baseline reference images acquired prior to sonication. At the end of the experiment, the animals were sacrificed for macroscopic and microscopic examinations of the kidney, liver and skin. The standard deviation of the temperature measured prior to heating in the sonicated area was approximately $1^{\circ} \mathrm{C}$ in kidney and liver, and $2.5^{\circ} \mathrm{C}$ near the skin. The maximum temperature rise was $30^{\circ} \mathrm{C}$ for a sonication of $1.2 \mathrm{MHz}$ in the liver over $15 \mathrm{~s}$ at $300 \mathrm{~W}$. The thermal dose reached the lethal threshold (240CEM 43 ) in two of six cases in the kidney and four of eight cases in the liver, but remained below this value in skin regions in the beam path. These findings were in agreement with histological analysis. Volumetric thermometry allows real-time monitoring of the temperature at the target location in liver and kidney, as well as in surrounding tissues. Thermal ablation was more difficult to achieve in renal than in hepatic tissue even using higher acoustic energy, probably because of a more efficient heat evacuation in the kidney by perfusion.
\end{abstract}

Keywords: MRI thermometry; high-intensity focused ultrasound (HIFU); liver; kidney; ablation

\section{INTRODUCTION}

Surgery remains the therapeutic approach of choice for liver and kidney tumours, although alternative minimally or noninvasive therapies are rapidly gaining popularity. The use of a local temperature increase by a physical heat source, such as a laser, radiofrequency $(\mathrm{RF})$, microwave (MW) or high-intensity focused ultrasound (HIFU), allows tumour destruction whilst preserving adjacent healthy tissues.

Noninvasive thermal therapies with HIFU have been successfully tested in the clinic for uterine fibroid ablation (1) and prostate (2). Leslie et al. (3) and Zhang et al. (4) have recently reported encouraging results on the HIFU treatment of large-dimension liver metastasis and hepatocellular carcinoma located at the vicinity of major hepatic veins. However, complete tumour necrosis was obtained in only one-half of the treated patients and no quantitative real-time monitoring of the temperature distribution was proposed.

The combination of local heating by HIFU with precise and noninvasive spatial mapping of the local temperature distribution during the procedure offers the possibility for the optimisation of this noninvasive therapy. Among the imaging modalities available in the clinical environment, MRI has been suggested as the method of choice, as it allows for noninvasive thermometry $(5,6)$ and offers excellent contrast in soft tissue for target visualisation and post-procedure follow-up. In addition, the calculation of the accumulated thermal dose (TD) from dynamic

\footnotetext{
* Correspondence to: B. Quesson, Laboratoire d'Imagerie Moleculaire et Fonctionnelle, UMR-CNRS 5231, Université Victor Segalen-Bordeaux 2, 146 Rue Leo Saignat, 33076 Bordeaux Cedex, France.

E-mail: b.quesson@imf.u-bordeaux2.fr

a B. Quesson, C. Laurent, G. Maclair, B. D. de Senneville, M. Ries, T. Carteret, C. T. Moonen Laboratoire d'Imagerie Moleculaire et Fonctionnelle, UMR 5231 CNRS/ Université Victor Segalen Bordeaux 2, Bordeaux, France

b C. Laurent Département de Chirurgie Digestive, Hôpital Saint André, Université Victor Segalen-Bordeaux 2, Bordeaux, France

c C. Mougenot Philips Healthcare, Suresne, France

d A. Rullier Département d'Anatomopathologie, Hôpital Pellegrin, Université Victor Segalen-Bordeaux 2, Bordeaux, France
}

Abbreviations used: bpm, beats per minute; CEM43, equivalent minutes at $43^{\circ} \mathrm{C}$; EPI, echo-planar imaging; HES, haematoxylin, eosin and safran; HIFU, high-intensity focused ultrasound; $M W$, microwave; $P R F$, proton resonance frequency; $R F$, radiofrequency; $R O I$, region of interest; $S N R$, signal-to-noise ratio; TD, thermal dose. 
temperature images can serve as an endpoint for the therapeutic procedure (7). MRI temperature imaging based on the measurement of the local variation of the frequency of the MR signal with temperature (so-called 'proton resonance frequency' shift, PRF shift) allows for the quantitative monitoring of local temperature evolution during HIFU ablation. However, precise monitoring of the temperature distribution remains difficult in abdominal organs because of the presence of motion and associated magnetic susceptibility changes. Moreover, the presence of blood flow in the kidney and liver constitutes one of the main sources of therapeutic failure, as it evacuates the heat outside the targeted region (8). MRI thermometry of these organs requires a high temporal resolution to correct for motion-related artefacts, to measure the displacement of the target and to precisely calculate TD. In addition, as the transducer is located outside the body, the monitoring of temperature should include the quantitative measurement of the temperature increase in adjacent healthy tissue located in the near field (skin and tissues between transducer and HIFU focal point) and far field (deep tissues located in the beam path behind the focal point).

To overcome the problem of organ displacement for temperature mapping, it has been suggested that the acquisition of MR images should be synchronised with the respiratory cycle (9) for monitoring long-duration (several minutes) RF ablation in the liver (10). Previous studies describing MR-guided HIFU ablation in liver and kidney have been presented, but with temperature monitoring $(11,12)$ limited to a single slice acquired at rather low temporal resolution $(6 \mathrm{~s})$. Therefore, the added value of temperature monitoring for predicting the resulting thermal lesion was limited. Few studies have presented quantitative images or the evolution of temperature. Recent developments in rapid acquisition techniques and parallel imaging capabilities of modern MRI scanners allow for a significant reduction in the acquisition time. Moreover, image processing for motion compensation has recently been developed (13), opening up the possibility for the development of MR-guided HIFU in abdominal organs, taking into account the organ position in the sonication strategy (14).

The objectives of this work were as follows: (i) to demonstrate the possibility to perform rapid and volumetric temperature monitoring of HIFU ablations in the kidney, liver and surrounding tissues; (ii) to evaluate the precision of thermometry with the help of an image-based motion compensation technique; (iii) to perform MR-guided HIFU ablations in the kidney and liver of pigs to evaluate the local temperature elevation and the resulting TD as a function of the acoustic energy.

\section{MATERIALS AND METHODS}

\section{Animals}

The animal experiments were conducted in agreement with French law on animal experimentation and in compliance with the rules for animal care and use of the CNRS and Universite Victor Segalen-Bordeaux 2, Bordeaux, France. All MR-guided HIFU experiments were performed in vivo on pigs. Five animals, weighing $40-50 \mathrm{~kg}$, were anaesthetised by intramuscular injection of propofol ( $10 \mathrm{~mL}$; Schering-Plough, Levallois-Perret, France). A 20-gauge intravenous catheter was then inserted into a dorsal ear vein and propofol was administered $(1 \mathrm{~mL} / \mathrm{min})$ to allow intubation with a 7-mm endotracheal tube. The abdomen and back of the pig were shaved, and hair removal cream was applied to remove residual hair, in order to ensure good acoustic coupling for the efficient propagation of HIFU pressure waves towards the liver or kidney. The animals were then transferred to the MR-compatible HIFU platform (see below for details) in the decubitus position for ablation in the kidney and in the prone position for ablation in the liver. All animals were maintained under assisted ventilation (respirator paraPac; ResMed SA, Saint Priest, France) during the procedure, with insufflation of $400 \mathrm{~mL}$ $\left(45 \% \mathrm{O}_{2}\right)$ at a frequency of 20 beats per minute (bpm). Anaesthesia was maintained with continuous intravenous injection of propofol $(40 \mathrm{~mL} / \mathrm{h})$ and ketamine $(10 \mathrm{~mL} / \mathrm{h}$; Merial, Lyon, France). Vital parameters, including body temperature and cardiac frequency, were monitored continuously. After completion of the MR-guided HIFU experiments, the animals were rapidly transferred to the experimental surgery laboratory (DETERCA, Université Victor Segalen-Bordeaux 2, Bordeaux, France), euthanised by intravenous injection of pentobarbital (CEVA Santé Animale, Libourne, France) and dissected for histology. The liver, kidneys and skin were removed, fixed in $10 \%$ buffered formalin for 7 days and analysed by the histology laboratory of Hospital Pellegrin, Bordeaux France.

\section{MR-compatible HIFU platform}

The thermal ablation experiments were performed with a Sonalleve MR-HIFU platform (Philips Healthcare, Vantaa, Finland), integrated in a 1.5 TAchieva-Intera MRI. This therapeutic platform comprised a table top containing a 256-element (operating frequency, 1.2 MHz) HIFU transducer (Imasonic, Besançon, France) mechanically positioned (three translations and two angulations) inside a tank of degassed water $\left(2 \mathrm{ppm} \mathrm{O}_{2}\right)$. The transducer radius and aperture were $120 \mathrm{~mm}$ and $126 \mathrm{~mm}$, respectively, creating an ellipsoidal focal point (pressure field full width at half-maximum, $1 \times 1 \times 7 \mathrm{~mm}^{3}$ ). The focal point position was modified electronically by adjusting the phases of the 256 output voltages of the generator cabinet. A thin circular membrane located on the top part of the water tank allowed the propagation of the HIFU beam towards the targeted region. To ensure correct ultrasound coupling, a gel pad was inserted between the animal and the membrane, with a mixture of degassed water and echographic gel to avoid the presence of air bubbles. A dedicated receiver coil was also integrated in this therapeutic device for MRI. The transducer position and focal point trajectory, together with the sonication parameters (amplitude, duration), were adjusted by the operator from a dedicated console located at the vicinity of the MRI console.

\section{Positioning of the transducer and sonication parameters}

Sonications within the lower part of the kidney were performed using an acoustic window inferior to the rib cage and lateral to the vertebral bodies, whereas sonications in the liver were performed using an acoustic window inferior to the sternum, into the right and left inferior lobes of the liver. Multi-planar anatomical images were acquired to verify the correct positioning of the targeted organ relative to the transducer and to check for the absence of air bubbles in the HIFU beam path. A set of balanced rapid gradient echo images (TE/TR $=1.56 / 3.02 \mathrm{~ms}$; Flip Angle $=60^{\circ}$, Field of view $=400 \mathrm{~mm}$; matrix size $=208 \times 238$ reconstructed to $256 \times 256$; slice thickness $=5 \mathrm{~mm}$; gap $=2 \mathrm{~mm}$; 22 slices) was acquired in horizontal and vertical planes to verify the relative positions of the transducer and the targeted area. 
From these images, the offsets in the position of the transducer were determined with the standard image analysis tools of the MRI console, and the transducer was repositioned if necessary. The distance between the target and the transducer was systematically set to $11.5 \mathrm{~cm}$. These acquisitions were repeated to check the final transducer position and to verify the absence of possible problematic anatomical structures (intestinal bowel, stomach, bones and lungs) within the HIFU beam path. For both organs, several acoustic powers and sonication durations were tested in order to evaluate the influence of the sonication conditions on the temperature evolution and resulting TD with rapid and volumetric MRI thermometry.

\section{MR thermometry acquisition and processing}

MR thermometry was performed before, during and after HIFU sonication with the PRF shift method. In order to provide volumetric and rapid thermometry, the acquisition sequence was a multi-slice $(n=5$, with a slice thickness of $6 \mathrm{~mm})$, gradient-recalled, single-shot, echo-planar imaging (EPI), with an effective echo time of $34 \mathrm{~ms}$, an echo train length of $61 \mathrm{~ms}$, a repetition time of $80 \mathrm{~ms}$, a flip angle of $30^{\circ}$, a field of view of $210 \times 300 \mathrm{~mm}$ and an acquisition matrix of $96 \times 112$, reconstructed to $112 \times 112$, leading to a nominal in-plane image resolution of $2.2 \times 2.7 \mathrm{~mm}^{2}$. A 121 binomial water-selective excitation was used to avoid lipid signals. Four images were acquired in the horizontal plane, with three adjacent slices centred on the focus of the HIFU beam and one slice located at the skin-gel interface. The fifth slice was positioned perpendicular to the four other slices to visualise the propagation of the HIFU beam through the organs, with the frequency encoding in the head-feet direction. Two saturation slabs were also added to avoid fold over artefacts. The image volume was acquired continuously with an update frequency of $2.5 \mathrm{~Hz}$.

Magnitude and phase images were reconstructed by the MRI system and streamed in real time to a separate workstation (dual processor Intel $3.1 \mathrm{GHz}$ Penryn, four cores, 8 GB RAM). Temperature images were calculated and displayed from in-house-developed software ('RealTI') written in C++ and IDL (ITT, Visual Information Solutions, Boulder, CO, USA). A small region of interest (ROI) of $5 \times 5$ pixels was selected outside the heated region on each slice to correct for apparent temperature drift in time. TD maps were also computed online using the Sapareto equation (15), taking the rectal temperature measured before each sonication as the reference temperature. The TD threshold for considering complete ablation was set to $240 \mathrm{~min}$ at a temperature of $43^{\circ} \mathrm{C}$, and TD values were calculated in equivalent minutes at this temperature $\left(\mathrm{CEM}_{43}\right)$.

For the liver, magnitude and phase images were processed to compensate for motion artefacts in temperature maps. Images acquired before sonication were stored in a reference atlas and used to compensate for the influence of motion on images acquired during sonication. The details of the correction algorithm can be found elsewhere (16). This correction was performed offline, except for the last animal for which online motion correction was implemented.

\section{Histological procedure}

After fixation, each organ (liver or kidney) or tissue (skin and muscle) was serially sliced to map thermal lesions. All slices were photographed before the selection of injured and surrounding normal tissue. Samples were formalin-fixed and paraffinembedded before examination by light microscopy. Analysis was performed after haematoxylin, eosin and safran (HES) staining in a blind fashion by the pathologist (AR) in order to describe the characteristics of the thermal lesions: localisation, size, aspect of injured tissue (haemorrhage, necrosis). The analysis of skin samples was also performed in order to look for potential alterations of the extracellular matrix. Refringence homogeneity of cutaneous fibrillar collagen was estimated by polarised light microscopy. Sections were examined with an Eclipse E400 Nikon microscope (Nikon, Bordeaux, France) at both $\times 100$ and $\times 200$ magnifications.

\section{RESULTS}

All sonications were successfully performed and a temperature increase was clearly identified in the temperature images. The body temperature was similar for each animal, with a typical maximal variation of $-2^{\circ} \mathrm{C}$ during anaesthesia. A representative example of the MR temperature and corresponding TD images of the kidney acquired during a 15-s HIFU sonication at an acoustic power of $300 \mathrm{~W}$ is shown in Fig. 1. A local temperature elevation is visible on the three sagittal slices, as well as on the coronal slice where the conical HIFU beam shape is visualised. The region with increased temperature appears larger on the sagittal slice positioned on the skin, and corresponds to the intersection of the HIFU cone outside the focal region. However, as expected, the maximal temperature increase in the near field is lower than that observed in the kidney. Calculation of TD for each slice shows that the value of $240 \mathrm{CEM}_{43}$ was reached in several pixels within the kidney in both sagittal and coronal slices. In contrast, no pixels in the slice located at the skin reached this value.

Figure 2a shows the temporal evolution of the temperature, measured at a sampling rate of $2.5 \mathrm{~Hz}$, at the focal point (central slice of the three sagittal slices positioned in the kidney) for three different HIFU sonications performed at the same location. The sonication parameters were $10 \mathrm{~s}$ at $100 \mathrm{~W}, 15 \mathrm{~s}$ at $200 \mathrm{~W}$ and $15 \mathrm{~s}$ at $300 \mathrm{~W}$, leading to total energy depositions of 1, 3 and $4.5 \mathrm{~kJ}$, respectively. As expected, the temperature increases with higher energy deposition. The standard deviation of the temperature measurement calculated from the 100 initial dynamics acquired prior to sonication was $0.68^{\circ} \mathrm{C}$ in the hottest pixel located at the intersection of the orthogonal slices. This value was similar to the measured value $\left(0.66^{\circ} \mathrm{C}\right)$ in the same slice, in a pixel located in the kidney but outside the cross-section of the orthogonal slices, indicative of a minor influence of partial saturation effects. At $200 \mathrm{~W}$ and $300 \mathrm{~W}$ during $15 \mathrm{~s}$, the peak temperature elevations were $17^{\circ} \mathrm{C}$ and $23^{\circ} \mathrm{C}$. Following HIFU application, the temperature decreased to half of the maximal increase within $10 \mathrm{~s}$ and $12 \mathrm{~s}$ for $200 \mathrm{~W}$ and $300 \mathrm{~W}$ applied HIFU power, respectively. In order to evaluate the potential change in standard deviation of the temperature during heating, the temperature curve corresponding to a heating of $300 \mathrm{~W}$ during $15 \mathrm{~s}$ (see Fig. 2a, b) was smoothed (boxcar average over seven adjacent time points) and subtracted from the original curve. As the temperature evolution is slow relative to the temporal resolution of the proposed sequence, this difference is mainly sensitive to the variation of the standard deviation of the temperature. The standard deviation of the difference measured over 20 successive points prior to heating was $0.49^{\circ} \mathrm{C}$, and increased to $1.21^{\circ} \mathrm{C}$ when the temperature reached its maximal value. 

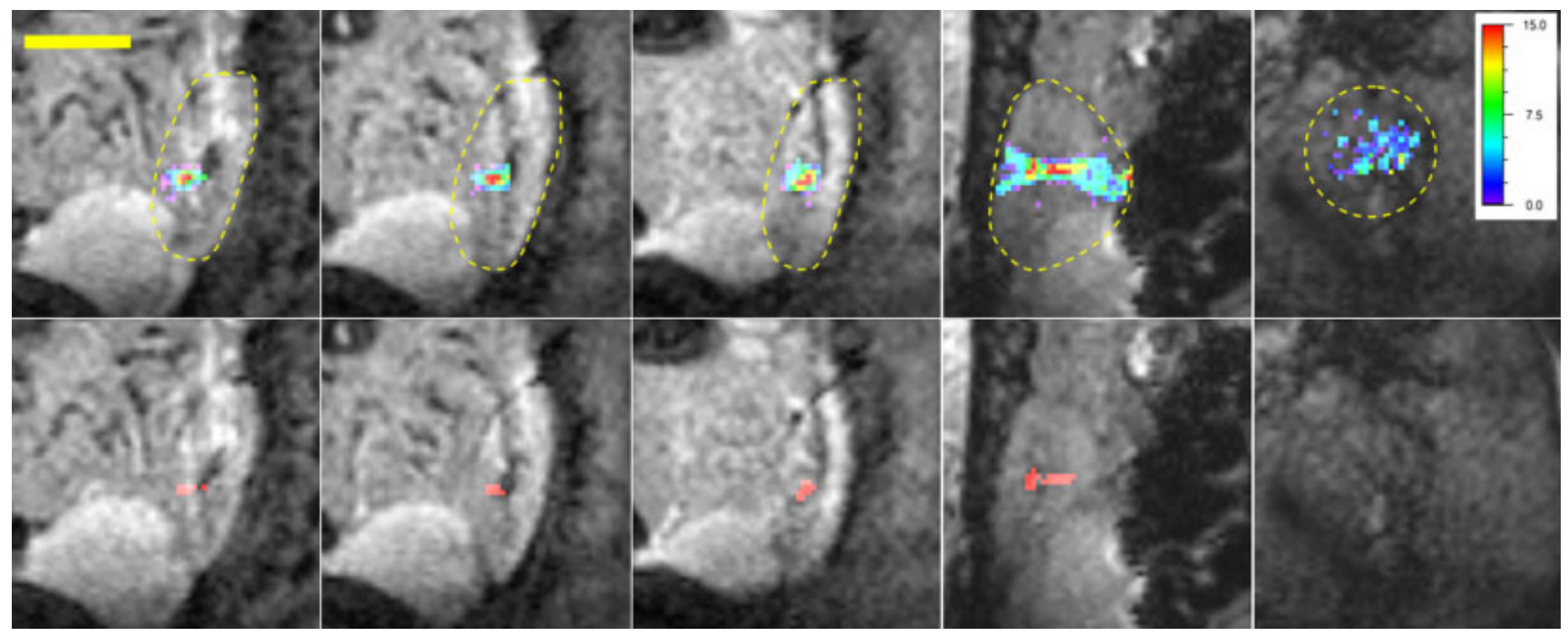

Figure 1. Temperature (top row, with colour scale on the right indicating the temperature change) and thermal dose [bottom row, with pixels coloured red corresponding to a thermal dose of $240 \mathrm{CEM}_{43}\left(\mathrm{CEM}_{43}\right.$, equivalent minutes at $\left.43^{\circ} \mathrm{C}\right)$ and above] images during high-intensity focused ultrasound (HIFU) ablation of the kidney. Left to right: three sagittal slices centred in the kidney, one coronal slice centred in the kidney and one sagittal slice located near the skin. The broken contours in the temperature images show the selected regions of interest (ROls) for displaying the temperature data. The horizontal yellow bar in the top left image represents $5 \mathrm{~cm}$. The temperature images display the temperature distribution measured at the end of the HIFU sonication and the thermal dose images are the final images in the time series.

Figure $2 \mathrm{~b}$ shows a comparison of the temperature increase for the $300-W$ sonication at the focal point and in the skin for a single pixel. The data correspond to the MR temperature and TD shown in Fig. 1. The standard deviation of the thermometry in the skin was $1.8^{\circ} \mathrm{C}$, because of a lower signal-to-noise ratio (SNR), in part caused by fat suppression of this anatomical region containing a high percentage of fat. The skin temperature showed a much lower increase than at the focal point $\left(6^{\circ} \mathrm{C}\right.$ relative to $\left.23^{\circ} \mathrm{C}\right)$, and remained nearly constant during the observation period (1 $\mathrm{min}$ $20 \mathrm{~s})$ of MR thermometry.

Figure $2 c$ shows a comparison of the temporal evolution of TD in the focal point within the kidney and in the skin. TD in the kidney exceeded the $240 \mathrm{CEM}_{43}$ value by a factor of 20 during the heating period, whereas it remained below $1 \%$ of this threshold in the skin. As the temperature in the kidney returned to its initial value during the observation period, TD in the kidney remained constant after it reached the threshold dose (at $55 \mathrm{~s}$ ), whereas TD in the skin continued to increase slightly because of the constant value of the temperature observed in Fig. $2 b$.

The analysis of the precision of thermometry in the liver with motion correction is presented in Fig. 3. The analysis of the distribution of the standard deviation values of temperature in the selected ROls in the liver revealed that $75 \%$ of the pixels displayed a standard deviation of the temperature lower than $2{ }^{\circ} \mathrm{C}$ for each slice. However, the standard deviation at the upper border of the liver exceeded $4{ }^{\circ} \mathrm{C}$ (pixels coloured in green and red). The standard deviation of the temperature in the skin (data not shown) was less than $2.60^{\circ} \mathrm{C}$ in $90 \%$ of the pixels, located in a circular $\mathrm{ROI}$ with a radius of 12 pixels surrounding the sonicated area, with maximal temperature standard deviation values of $38^{\circ} \mathrm{C}$ corresponding to a limited number of pixels with very low SNR. Similar results were found for each animal.

Figure 4 shows a HIFU ablation in the liver performed with motion compensation. A local temperature increase is observed in each slice with the hottest spot centred in the liver (Fig. 4a). The standard deviation prior to heating (see Fig. 3b) in the sonicated area was $0.9^{\circ} \mathrm{C}$ in the liver (slices $1-4$ ) and $2.5^{\circ} \mathrm{C}$ in the skin (average value within a circular ROI with a radius of 12 pixels covering the beam path). Similar to the kidney, the temperature increase was sufficient to reach the $240 \mathrm{CEM}_{43}$ threshold in the liver, but not in the skin. Analysis of the standard deviation of the temperature in the focal point, with identical processing as for the kidney (difference between experimental temperature and smoothed temperature), resulted in a standard deviation value of $0.55^{\circ} \mathrm{C}$ before heating and $0.90^{\circ} \mathrm{C}$ when the temperature reached its maximal value.

Figure 5 shows the temporal evolution of the temperature at the focal point (central slice of the coronal stack) without and with motion correction. The oscillations of the temperature observed without motion correction (Fig. 5a) are compensated after correction (Fig. 5b). The corrected temperature elevation increased to $20^{\circ} \mathrm{C}$ and returned to its initial value after HIFU application. Some residual oscillations can be seen during the cooling period, but with a maximal amplitude of $1^{\circ} \mathrm{C}$ only, in the same range as the standard deviation of the temperature estimate.

Table 1 summarises the results of the 14 different sonications performed in the kidney $(n=6)$ and liver $(n=8)$. Each sonication was performed with different HIFU acoustic power or sonication duration to modify the effective energy deposition within the targeted organ. One sonication at constant power with continuous electronic displacement of the focal beam was tested on the liver (last row), consisting of two consecutive concentric circular trajectories of 4 and $8 \mathrm{~mm}$ in diameter with respective durations of 17 and $10 \mathrm{~s}$ [as suggested in ref. (17)]. Analysis of the standard deviation of the temperature at the focal point (see above) resulted in a standard deviation of $0.78^{\circ} \mathrm{C}$ before heating and $0.75^{\circ} \mathrm{C}$ when the temperature reached its maximal value.

The second column of Table 1 gives the effective sonication duration, which was shorter than the planned value in four cases. The reason for this difference is a hardware security check of the 

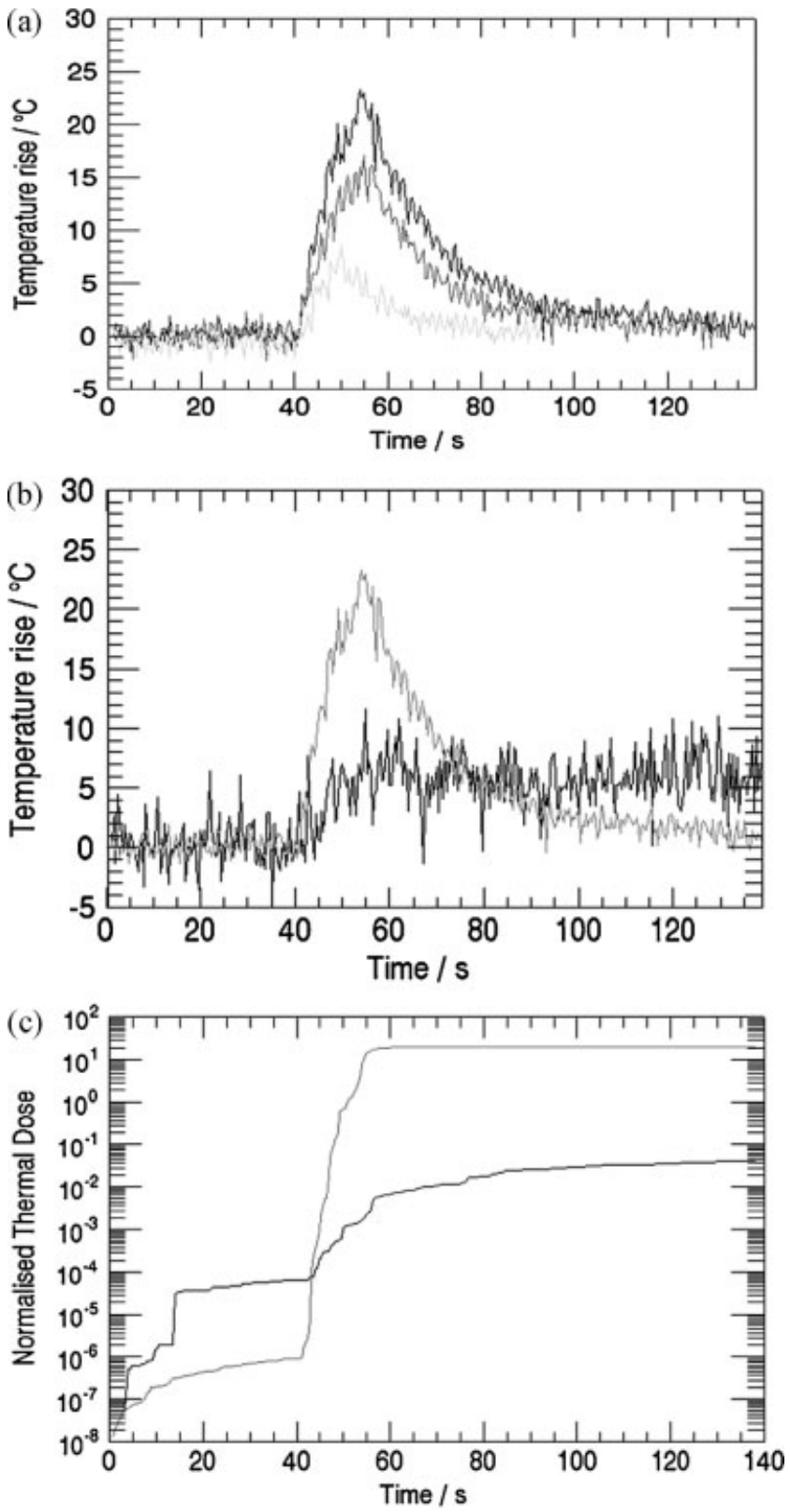

Figure 2. Temporal evolution of temperature and thermal dose in the kidney. The top graph (a) shows the temperature increase in a single voxel located at the focal point for three different sonication parameters: $100 \mathrm{~W}$ for $10 \mathrm{~s}$ (light grey), $200 \mathrm{~W}$ for $15 \mathrm{~s}$ (dark grey) and $300 \mathrm{~W}$ for $15 \mathrm{~s}$ (black). The graph in the middle (b) shows the temperature evolution at the focal point (light grey) and in a single pixel located at the centre of the heated region near the skin (black) for a sonication at $300 \mathrm{~W}$ for $15 \mathrm{~s}$. The bottom graph (c) shows the temporal evolution of the thermal dose (decimal logarithm scale) for the temperature evolutions displayed in (b). For convenience, the equivalent minutes were normalised to the selected thermal dose threshold $\left[10^{\circ}(=1)\right.$ represents the threshold of $240 \mathrm{CEM}_{43}$ $\left(\mathrm{CEM}_{43}\right.$, equivalent minutes at $\left.\left.43^{\circ} \mathrm{C}\right)\right]$.

HIFU platform, halting the sonication when the transducer temperature exceeds a predefined threshold. As a consequence, the total transmitted acoustic energy (third column) was lower than the expected value, and ranged from 1 to $4.5 \mathrm{~kJ}$ for the kidney and from 0.8 to $4.86 \mathrm{~kJ}$ for the liver. For both organs, the standard deviation of the temperature ranged between 0.67 and $1.2^{\circ} \mathrm{C}$. These values were measured prior to HIFU deposition in the pixel located at the HIFU focal point and without spatial averaging. The maximal temperature increase ranged from 10 to $23^{\circ} \mathrm{C}$ in the kidney and from 4.8 to $30^{\circ} \mathrm{C}$ in the liver. No motion was observed in the kidneys. In contrast, a displacement of the liver was observed during each sonication and required a systematic application of motion correction in thermal maps.

For fixed HIFU focal point sonications, the required acoustic energy to reach $20^{\circ} \mathrm{C}$ (or more) temperature increase was higher in the kidney $(4.2-4.5 \mathrm{~kJ})$ than in the liver $(2.7-3.6 \mathrm{~kJ})$. For one sonication (15 s at $200 \mathrm{~W}$ ) in the liver, the maximal temperature increase was only $15.5^{\circ} \mathrm{C}$. However, this experiment was performed under spontaneous breathing conditions (accidental deactivation of the mechanical ventilation), which resulted in more rapid and larger displacements of the organ relative to sonications performed under mechanical ventilation. A second sonication with identical parameters was repeated at $3 \mathrm{~mm}$ from the previous target location during ventilation, resulting in a temperature increase of $25^{\circ} \mathrm{C}$. The maximal temperature increase obtained in the liver was $30^{\circ} \mathrm{C}$ for an acoustic energy of $3.6 \mathrm{~kJ}$. It should be noted that such temperature increases could not be reached in the kidney, even for a higher value of energy deposition ( $4.5 \mathrm{~kJ}$ ). TD exceeded the $240 \mathrm{CEM}_{43}$ threshold in two of six cases in the kidney and four of eight cases in the liver. For kidney, even for high values of transmitted energy (higher or equal to $4.2 \mathrm{~kJ}$ ), the efficiency of the sonication was limited as TD exceeded the threshold in only two of four cases. For liver, energy levels higher than $2.7 \mathrm{~kJ}$ were sufficient to reach the threshold in four of five cases.

\section{Pathological analysis}

Macroscopic lesions were found in the kidney and liver for each case when TD reached or exceeded the $240 \mathrm{CEM}_{43}$ value (Table 1). The lesion size is reported in two dimensions. The precise three-dimensional estimation of the ablated volume could not be determined because of the limited dimensions of the thermal lesions with respect to the typical thickness $(2-3 \mathrm{~mm})$ of the slices after organ fixation. Thermal lesion patterns observed in the kidney and liver were quite similar, showing an intraparenchymatous nodular or elongated lesion $(6-17 \mathrm{~mm})$ with a dark rim and white centre (Fig. 6a, c). Microscopically, the white centre corresponded to necrotic or pre-necrotic tissue surrounded by a rim of haemorrhagic and pre-necrotic tissue (Fig. 6b, d). Cutaneous and subcutaneous tissues (adipose tissue and muscle) showed no damage for all cases (Fig. 6e, f). Examination by polarised light microscopy did not reveal any alterations of the extracellular matrix, confirming the absence of collateral damage in the skin under the different tested sonications.

\section{DISCUSSION}

The combination of fast temperature imaging with efficient motion compensation provides precise, volumetric and quantitative monitoring of temperature evolution within the targeted organ (kidney or liver) and the surrounding tissues during HIFU ablation. The temporal resolution of $400 \mathrm{~ms}$ per volume allowed for a noninvasive measurement of the local temperature distribution, which is of particular interest for highly perfused and/or mobile organs targets such as kidney and liver tumours.

The monitoring of the temperature in the liver was complicated by the presence of respiratory motion. The association of rapid temperature imaging with an efficient 


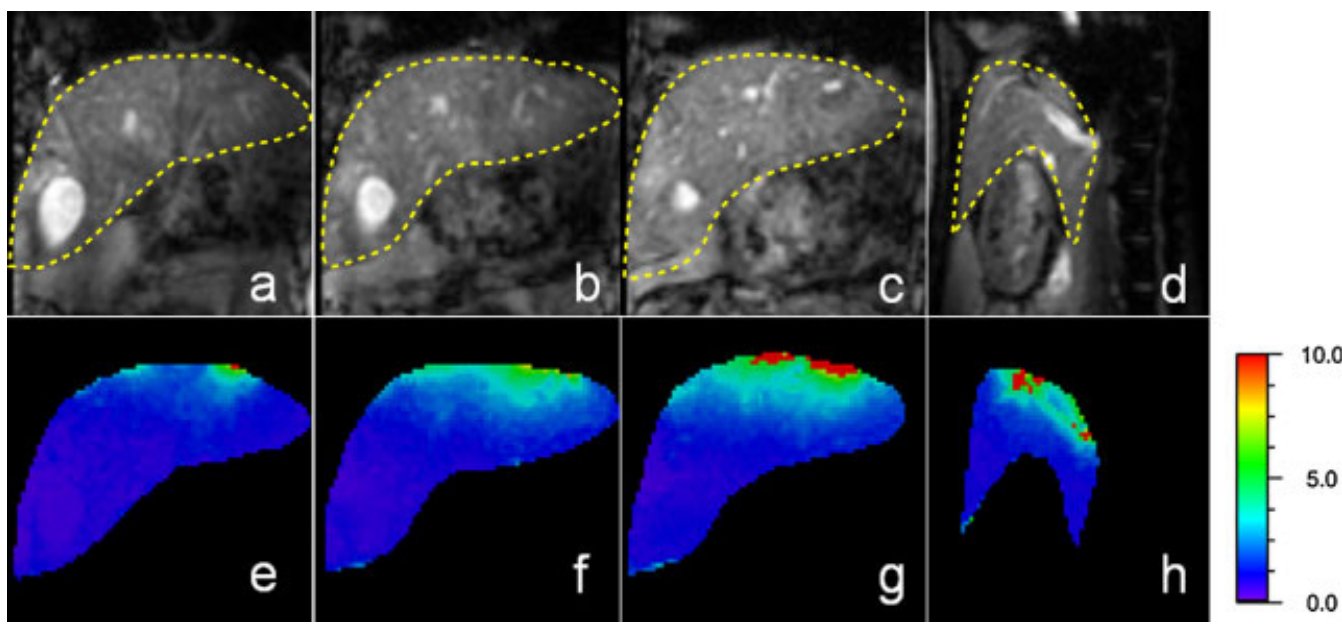

Figure 3. Analysis of the precision of the temperature measurement in the liver with motion correction and without high-intensity focused ultrasound (HIFU) ablation. Top row shows coronal (a-c) and sagittal (d) magnitude images and bottom row displays the corresponding maps (e-h) of the standard deviation of the temperature (the scale of the temperature standard deviation is indicated on the right). The broken contours superimposed on the magnitude images show the selected regions of interest (ROIs) for the analysis of the standard deviation of temperature within the liver.

motion compensation algorithm clearly improved the standard deviation of the thermal maps as, after correction, the median value of the standard deviation of the temperature was lower than $2^{\circ} \mathrm{C}$ for all slices and in the region of $1^{\circ} \mathrm{C}$ in the sonicated area. These values were in the same range as those reported by Rempp et al. (18) at $1.5 \mathrm{~T}$ in volunteers, but under respiratory-triggered acquisitions. With this precision of thermometry, even a moderate temperature increase of $4^{\circ} \mathrm{C}$ induced by low-energy deposition $(0.8 \mathrm{~kJ})$ could be clearly identified in temperature images. Therefore, nondestructive test sonications can be monitored to check the location of the HIFU beam in oncological applications, prior to sonications with higher acoustic energy. However, the precision of thermometry remains limited in the superior part of the liver, at the vicinity of the lung and heart, probably because of differences in local magnetic susceptibility at tissue interfaces and rapid cardiac motion. This adverse effect may also be attenuated by reducing the echo train duration of the single-shot EPI sequence with the use of parallel imaging. The gain in temporal resolution may then be exploited to increase the number of images in the multi-baseline reference dataset to resolve the influence of the cardiac motion in the pixels of the liver located near the heart. In addition, the shortening of the echo train duration and effective echo time should attenuate image distortions and lead to a higher SNR. However, these potential improvements given by parallel imaging could not be tested in the present work because of the limited number of elements of the receiver coil integrated in the HIFU platform. Other strategies have been proposed for the correction of temperature images (19) and interscan motion (20), and may provide alternative solutions to improve MR thermometry.

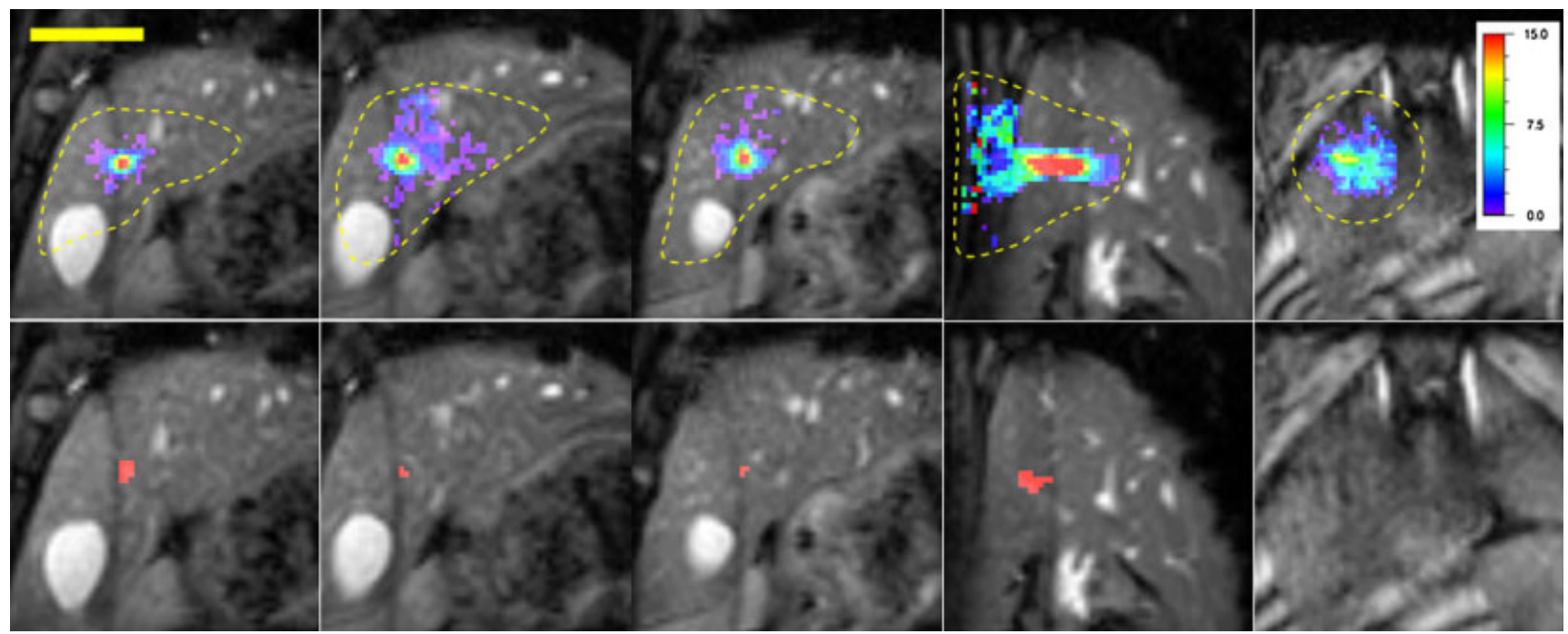

Figure 4. Temperature (top row) and thermal dose (bottom row) images of the liver during high-intensity focused ultrasound (HIFU). The temperature scale is displayed on the right. The pixels coloured in red correspond to a thermal dose of $240 \mathrm{CEM}_{43}$ and above $\left(\mathrm{CEM}_{43}\right.$, equivalent minutes at $\left.43^{\circ} \mathrm{C}\right)$. Left to right: three coronal slices centred around the target point, one sagittal slice centred in the target point and one slice located near the skin. The broken contours in the temperature images show the selected regions of interest (ROIs) for displaying the temperature data. The temperature images display the temperature distribution measured at the end of HIFU sonication, and the thermal dose images are the final images in the time series. 

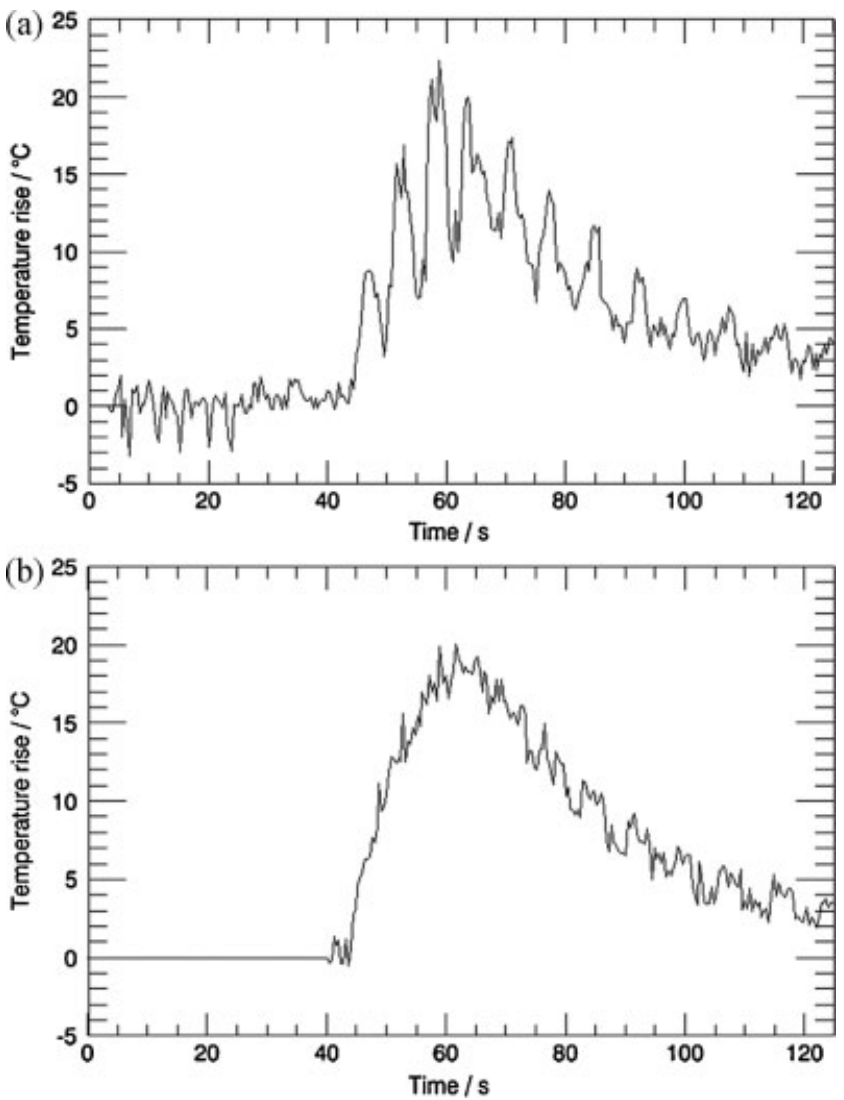

Figure 5. Temporal evolution of temperature in the liver at the focal point without (a) and with (b) motion correction. In (b), the magnitude and phase images during the first $40 \mathrm{~s}$ were stored in the atlas of reference images (no temperature estimate).

The temperature evolution in the kidney and liver displays an important temperature rise during sonication, followed by tissue cooling. During heating, the standard deviation of the temperature was increased by a factor of $2.63(1.21 / 0.49)$ for single-point heating in the kidney and $1.63(0.90 / 0.55)$ in the liver. This loss in precision of MR thermometry can be attributed to intravoxel dephasing because of the steep temperature gradients resulting from single-point heating, affecting the SNR of MR images acquired with short repetition times. However, for the experiment performed with two consecutive concentric circular trajectories, the standard deviation of the temperature was not altered. This indicates that the loss of precision depends mainly on the heating strategy, and that the volumetric heating approaches are preferable with respect to the accuracy of thermometry and estimation of TD. Recent results on MR-guided HIFU performed in vivo in pig muscle (17) showed that ablation sizes estimated from post-sonication follow-up images and histological analysis correlated with the dimensions of the apparent lesion on TD images with the same threshold value $\left(240 \mathrm{~min}\right.$ at $\left.43^{\circ} \mathrm{C}\right)$. This correlation was not performed in the present work because of the limited spatial resolution of MR thermometry with respect to the investigated sonication strategies, resulting in thermal lesions of limited size (Table 1).

As expected, the local temperature increases were found to be dependent on the acoustic power and sonication duration. Both liver and kidney required high acoustic power to reach the $240 \mathrm{CEM}_{43}$ threshold. However, thermal lesions were more difficult to achieve in kidney relative to liver, in agreement with studies by Daum et al. (11), who also reported thermal lesions of smaller size in the kidney than in the liver. This was attributed to differences in tissue thermal properties (i.e. absorption, perfusion and thermal conductivity). A higher perfusion has been reported in the kidney than in the liver (21), resulting in a more efficient heat evacuation during heating and cooling, and a lower TD. The results of the present study are in good agreement with this observation, as a higher energy was required to reach the $240 \mathrm{CEM}_{43}$ value in the kidney $(4.5 \mathrm{~kJ})$ than in the liver $(2.7 \mathrm{~kJ})$. The acoustic energy levels given in the present work are in the same range as those reported in the literature $(11,12)$. Kopelman et al. (12) reported high temperature increases (with an absolute maximal temperature ranging from 60 to $80^{\circ} \mathrm{C}$ ) in the liver with short-duration sonications (20 s) performed under breath-hold conditions. Daum et al. (11) reported temperature increases ranging from 15 to $40^{\circ} \mathrm{C}$ for sonications performed in pig kidney and liver under free-breathing conditions. In the latter study, histological analyses revealed a limited correspondence between lesions and MRI temperature imaging data. However, for both studies, MRI temperature monitoring was performed in a single slice at an update rate of $3.4 \mathrm{~s}$ or more, without quantitative analysis of the precision of thermometry $(11,12)$. In the present work, rapid and volumetric MRI thermometry was performed with an update rate of $400 \mathrm{~ms} /$ volume and high precision of the temperature estimate. All lesions observed in histological analysis were also identified in the TD maps.

For all experiments, the standard deviation of the temperature was higher near the skin relative to the target location, probably because of lower SNR, resulting from lipid suppression. As the temperature evolution is slow relative to the sampling frequency of the thermometry sequence $(2.5 \mathrm{~Hz})$, and the heating pattern of the skin covers a large area, a low-pass temporal and/or spatial filter could also be applied to temperature data to improve the precision of TD calculation in this region. The required high levels of acoustic power for thermal ablation of the kidney and liver increased the risk of inducing necrosis in adjacent healthy tissues (e.g. skin, intestine, bowel), and thus necessitates rapid temperature mapping. The temperature near the skin showed a lower increase relative to values reached in the liver and kidney, but remained nearly constant after the end of heating, as shown in Fig. $2 b$. This can be explained by the fact that temperature gradients are lower near the skin. In addition, perfusion is known to be lower in the skin relative to the kidney and liver (21). Therefore, temperature evolution can be expected to be mainly governed by heat diffusion. As a consequence, TD in the skin continued to increase after the end of heating, but never exceeded the $240 \mathrm{CEM}_{43}$ threshold.

Maximal sonication durations at high acoustic power were limited by the thermal security check of the transducer. Therefore, improving the HIFU hardware is a prerequisite for investigating alternative heating strategies based on long-duration sonications performed at multiple focal points (22) or in volumetric sonications $(17,23)$.

The implementation of online motion correction for thermometry opens up the possibility of improving the precision of temperature monitoring during HIFU ablation, and should allow for dynamic adjustment of the acoustic power and positioning of the HIFU focal point (24-26).

The volumetric coverage of the temperature imaging sequence allows the monitoring of the temperature at the targeted location and in the surrounding tissues. Moreover, fast updating of the thermal maps is essential for sonications 
Table 1. Summary of MR-guided high-intensity focused ultrasound (HIFU) experiments performed in kidney and liver as a function of the sonication conditions

\begin{tabular}{|c|c|c|c|c|c|c|}
\hline $\begin{array}{l}\text { Sonication } \\
\text { parameters (W, s) }\end{array}$ & $\begin{array}{c}\text { Effective } \\
\text { duration }^{a}(\mathrm{~s})\end{array}$ & $\begin{array}{c}\text { Deposited } \\
\text { energy }(k J)\end{array}$ & $\begin{array}{c}T \text { standard } \\
\text { deviation }{ }^{\mathrm{b}}\left({ }^{\circ} \mathrm{C}\right)\end{array}$ & $\begin{array}{c}\text { Max } T \\
\text { increase }\left({ }^{\circ} \mathrm{C}\right)\end{array}$ & $\begin{array}{l}\text { TD threshold } \\
\text { reached }\end{array}$ & $\begin{array}{l}\text { Lesion size }{ }^{c} \\
(\mathrm{~mm} \times \mathrm{mm})\end{array}$ \\
\hline \multicolumn{7}{|l|}{ Kidney } \\
\hline 100,10 & 10 & 1.0 & 0.85 & 10 & No & NA \\
\hline 200,15 & 15 & 3.0 & 0.67 & 18 & No & NA \\
\hline 300,15 & 15 & 4.5 & 0.68 & 23 & Yes & $9 \times 5$ \\
\hline 300,15 & 15 & 4.5 & 1.18 & 22 & Yes & $13 \times 3$ \\
\hline 300,15 & 15 & 4.5 & 0.9 & 17 & No & - \\
\hline 300,15 & 14 & 4.2 & 0.9 & 19.6 & No & - \\
\hline \multicolumn{7}{|l|}{ Liver } \\
\hline 80,10 & 10 & 0.8 & 0.9 & 4.8 & No & - \\
\hline 100,10 & 10 & 1.0 & 1.2 & 11 & No & - \\
\hline 120,15 & 15 & 1.8 & 1.1 & 11 & No & - \\
\hline $200,15^{\mathrm{d}}$ & 13.5 & 2.7 & 0.9 & 15.5 & No & - \\
\hline 200,15 & 13.5 & 2.7 & 0.9 & 25 & Yes & $12 \times 7$ \\
\hline $200,15^{\mathrm{e}}$ & 15 & 3.0 & 0.9 & 23 & Yes & $12 \times 6$ \\
\hline 300,15 & 12 & 3.6 & 1.1 & 30 & Yes & $14 \times 6$ \\
\hline $180,27^{f}$ & 27 & 4.86 & 1.2 & 22.5 & Yes & $17 \times 8$ \\
\hline \multicolumn{7}{|c|}{$\begin{array}{l}\text { NA, not available. } \\
\text { aEffective sonication duration because of halting of sonication on overheating of the transducer (internal thermal security check of the } \\
\text { transducer). } \\
\text { b Values obtained without sonication and rounded to the upper decimal. } \\
\text { cReported values were measured in the macroscopic slice displaying the larger thermal lesion area. } \\
\text { dexperiment performed under spontaneous breathing. } \\
\text { experiment performed with online motion correction. } \\
\text { f } \\
\text { fonication performed with two concentric circles of } 4 \mathrm{~mm} \text { in diameter for the inner circle }(17 \mathrm{~s} \text { duration) and } 8 \mathrm{~mm} \text { in diameter for the outer } \\
\text { circle (10 s duration). }\end{array}$} \\
\hline
\end{tabular}

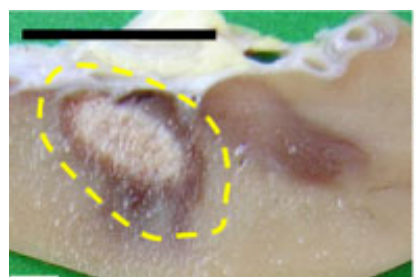

(a)

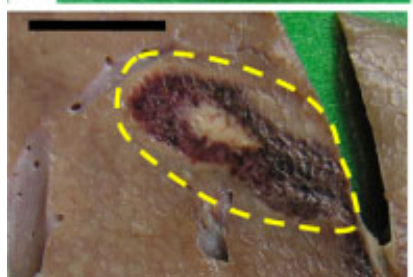

(c)
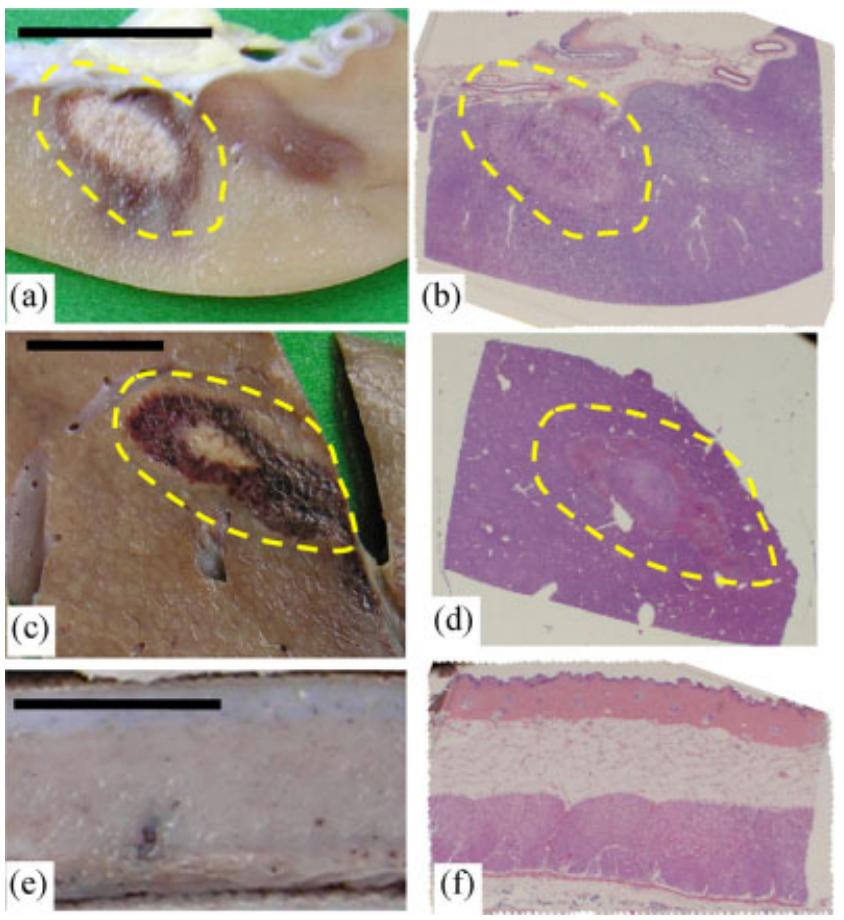

(b)

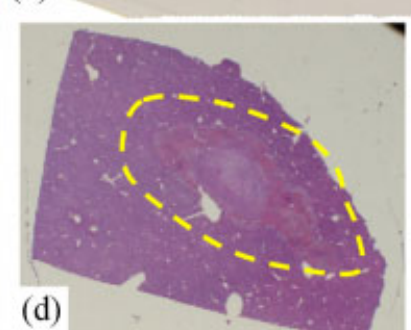

Figure 6. Macroscopic (left column) and haematoxylin, eosin and safran (HES)-stained (right column) images of the kidney $(a, b$, sonication at $300 \mathrm{~W}$ for $15 \mathrm{~s}$ ), liver (c, d, sonication at $200 \mathrm{~W}$ for $15 \mathrm{~s}$ ) and cutaneous and subcutaneous tissues ( $e, f$, same sonication as in a and b). The black horizontal bars represent $1 \mathrm{~cm}$. The lesions are surrounded by yellow broken contours in images (a)-(d). performed with such high acoustic intensities. The association of volumetric and rapid thermometry should help to avoid collateral damage and therefore increase the safety of the procedure for the patient.

\section{Acknowledgements}

The authors thank the 'Agence Nationale pour la Recherche', the 'Ligue Nationale Contre le Cancer' and the 'Fondation Innabiosanté' for financial support, and Philips Medical Systems, Vantaa, Finland for technical support.

\section{REFERENCES}

1. Fennessy FM, Tempany CM. A review of magnetic resonance imaging-guided focused ultrasound surgery of uterine fibroids. Top. Magn. Reson. Imaging, 2006; 17: 173-179.

2. Illing $R$, Chapman A. The clinical applications of high intensity focused ultrasound in the prostate. Int. J. Hyperthermia, 2007; 23: 183-191.

3. Leslie TA, Kennedy JE, Illing RO, Ter Haar GR, Wu F, Phillips RR, Friend PJ, Roberts IS, Cranston DW, Middleton MR. High-intensity focused ultrasound ablation of liver tumours: can radiological assessment predict the histological response? Br. J. Radiol. 2008; 81: 564-571.

4. Zhang L, Zhu H, Jin C, Zhou K, Li K, Su H, Chen W, Bai J, Wang Z. High-intensity focused ultrasound (HIFU): effective and safe therapy for hepatocellular carcinoma adjacent to major hepatic veins. Eur. Radiol. 2009; 19: 437-445.

5. Quesson B, de Zwart JA, Moonen CT. Magnetic resonance temperature imaging for guidance of thermotherapy. J. Magn. Reson. Imaging, 2000; 12: 525-533. 
6. Rieke V, Butts Pauly K. MR thermometry. J. Magn. Reson. Imaging, 2008; 27: 376-390.

7. Seror O, Lepetit-Coiffe M, Le Bail B, de Senneville BD, Trillaud H, Moonen C, Quesson B. Real time monitoring of radiofrequency ablation based on MR thermometry and thermal dose in the pig liver in vivo. Eur. Radiol. 2008; 18: 408-416.

8. Frericks BB, Ritz JP, Albrecht T, Valdeig S, Schenk A, Wolf KJ, Lehmann $K$. Influence of intrahepatic vessels on volume and shape of percutaneous thermal ablation zones: in vivo evaluation in a porcine model. Invest. Radiol. 2008; 43: 211-218.

9. Weidensteiner C, Kerioui N, Quesson B, de Senneville BD, Trillaud $H$, Moonen $C T$. Stability of real-time MR temperature mapping in healthy and diseased human liver. J. Magn. Reson. Imaging, 2004; 19: 438-446.

10. Lepetit-Coiffe M, Quesson B, Seror O, Dumont E, Le Bail B, Moonen CT, Trillaud $\mathrm{H}$. Real-time monitoring of radiofrequency ablation of rabbit liver by respiratory-gated quantitative temperature MRI. J. Magn. Reson. Imaging, 2006; 24: 152-159.

11. Daum DR, Smith NB, King R, Hynynen K. In vivo demonstration of noninvasive thermal surgery of the liver and kidney using an ultrasonic phased array. Ultrasound Med. Biol. 1999; 25: 1087-1098.

12. Kopelman D, Inbar Y, Hanannel A, Dank G, Freundlich D, Perel A, Castel D, Greenfeld A, Salomon T, Sareli M, Valeanu A, Papa M. Magnetic resonance-guided focused ultrasound surgery (MRgFUS). Four ablation treatments of a single canine hepatocellular adenoma. HPB (Oxford), 2006; 8: 292-298.

13. Denis de Senneville B, Quesson B, Moonen CT. Magnetic resonance temperature imaging. Int. J. Hyperthermia, 2005; 21: 515-531.

14. Denis de Senneville BD, Mougenot C, Moonen CT. Real-time adaptive methods for treatment of mobile organs by MRI-controlled highintensity focused ultrasound. Magn. Reson. Med. 2007; 57: 319-330.

15. Sapareto SA, Dewey WC. Thermal dose determination in cancer therapy. Int. J. Radiat. Oncol. Biol. Phys. 1984; 10: 787-800.

16. Denis de Senneville B, Quesson B, Desbarats P, Salomir R, Palussière J, Moonen C. Atlas based motion correction for on line MR temperature mapping. Proceedings of IEEE International Conference on Image Processing (ICIP), Singapore, 2004; 2571-2574.
17. Köhler MO, Mougenot C, Quesson B, Enholm J, Le Bail B, Laurent C, Moonen CT, Ehnholm GJ. Volumetric HIFU ablation under 3D guidance of rapid MRI thermometry. Med. Phys. 2009; 36: 3521-3535.

18. Rempp H, Martirosian P, Boss A, Clasen S, Kickhefel A, Kraiger M, Schraml C, Claussen C, Pereira P, Schick F. MR temperature monitoring applying the proton resonance frequency method in liver and kidney at 0.2 and $1.5 \mathrm{~T}$ : segment-specific attainable precision and breathing influence. Magma, 2008; 21: 333-343.

19. Rieke V, Kinsey AM, Ross AB, Nau WH, Diederich CJ, Sommer G, Pauly KB. Referenceless MR thermometry for monitoring thermal ablation in the prostate. IEEE Trans. Med. Imaging, 2007; 26: 813-821.

20. Suprijanto S, Vogel MW, Vos FM, Vrooman HA, Vossepoel AM. Interframe motion correction for MR thermometry. Med. Image Comput. Comput. Assist. Interv. 2005; 8: 580-588.

21. Cheng HL, Plewes DB. Tissue thermal conductivity by magnetic resonance thermometry and focused ultrasound heating. J. Magn. Reson. Imaging, 2002; 16: 598-609.

22. Fan X, Hynynen K. Ultrasound surgery using multiple sonications treatment time considerations. Ultrasound Med. Biol. 1996; 22: 471-482.

23. Palussiere J, Salomir R, Le Bail B, Fawaz R, Quesson B, Grenier N, Moonen CT. Feasibility of MR-guided focused ultrasound with realtime temperature mapping and continuous sonication for ablation of VX2 carcinoma in rabbit thigh. Magn. Reson. Med. 2003; 49: 89-98.

24. Aubry JF, Pernot M, Marquet F, Tanter M, Fink M. Transcostal highintensity-focused ultrasound: ex vivo adaptive focusing feasibility study. Phys. Med. Biol. 2008; 53: 2937-2951.

25. Chopra R, Baker N, Choy V, Boyes A, Tang K, Bradwell D, Bronskill MJ. MRI-compatible transurethral ultrasound system for the treatment of localized prostate cancer using rotational control. Med. Phys. 2008; 35: 1346-1357.

26. Mougenot C, Salomir R, Palussiere J, Grenier N, Moonen CT. Automatic spatial and temporal temperature control for MR-guided focused ultrasound using fast 3D MR thermometry and multispiral trajectory of the focal point. Magn. Reson. Med. 2004; 52: 1005-1015. 